\title{
Efeito anti-inflamatório do gengibre e possível via de sinalização
}

\section{Anti-inflammatory effect of ginger and possible signaling pathway}

\author{
Nichelle Antunes Vieira ${ }^{1}$; Fernanda Nogueira Tomiotto ${ }^{1}$; Gabriella Pasqual Melo ${ }^{1}$; \\ Marília Fernandes Manchope ${ }^{1}$; Nicole Ribeiro de Lima'; Gabriela Gonçalves de \\ Oliveira $^{2}$; Maria Angélica Ehara Watanabe ${ }^{3}$
}

\begin{abstract}
Resumo
O gengibre (Zingiber officinale Roscoe) vem sendo utilizado tanto na medicina natural tradicional quanto contemporânea, sendo descritos mais de 115 componentes do gengibre fresco e seco. Dentre esses componentes, os compostos fenólicos, gingerol e o shoagol, têm sido amplamente estudados apresentando diferentes propriedades destas moléculas como efeito antipirético, analgésico, inibidor da angiogênese, atividades imunomoduladoras, entre outras. Esta revisão tem como objetivo apresentar os componentes do gengibre assim como o seu efeito antagônico sobre o lipopolissacarídeo (LPS), propriedade anti- inflamatória e possível via de sinalização envolvida. $\mathrm{O}$ efeito do gengibre sobre o LPS consiste na inibição da produção de Interleucina 12 (IL -12), resultando em menor ativação de macrófagos induzidos pelo LPS; diminuição na expressão de moléculas co-estimulatórias e expressão de MHC classe II e diminuição de Interleucina 2 (IL-2), suprimindo a ativação e proliferação de células T CD4+. A propriedade anti-inflamatória do composto está relacionada com a capacidade de modular o linfócito $\mathrm{T}$, de forma a inibir a eosinofilia, reduzir a quantidade de mastócitos, inibir a liberação de IL-4 e reduzir a resposta Th2. Além disso, pode ocorrer inibição da translocação da subunidade p65 para o núcleo e inibição da fosforilação do complexo IkB- $\alpha$, culminando com a diminuição dos níveis de COX-2. Através deste estudo, podemos concluir que componentes do gengibre podem atuar antagonicamente sobre o efeito de ativação do LPS em macrófagos, conduzindo um efeito imunomodulador e anti-inflamatório, no qual a possível via de sinalização afetada seja do NFkB com envolvimento das MAPquinases.
\end{abstract}

Palavras-Chave: Zingiber officinale Roscoe. Gingerol. Shogaol. Inflamação

\begin{abstract}
Ginger (Zingiber officinale Roscoe) has been used in both traditional and contemporary natural medicine, with over 115 described components of fresh and dried ginger. Among these components, the phenolic compounds: gingerol and shoagol have been broadly studied and these molecules have different properties, such as antipyretic, analgesic, angiogenesis inhibitor, immunomodulatory activities, among others. This review aims to present the components of ginger as well as its antagonistic effect on LPS, anti - inflammatory property and possible signaling pathway involved. The effect of ginger on lipopolysaccharide (LPS) consists in inhibiting the production of interleukin 12 (IL-12), resulting in reduced activation of macrophages induced by LPS; decreased expression of co-stimulatory molecules and MHC class II expression and reduced IL-2, impairing the activation and proliferation of CD4 + $\mathrm{T}$ cells. The anti-inflammatory property of the compound is associated with the ability to modulate $\mathrm{T}$ lymphocyte, in order to inhibit eosinophilia, reducing the amount of mast cells and inhibit the release

${ }^{1}$ Graduanda do Curso de Biomedicina, Universidade Estadual de Londrina (UEL) - PR

${ }^{2}$ Doutora em Patologia Experimental. Aluna de Pós-doutorado na Faculdade de Medicina de Ribeirão Preto - USP.

3 Doutora em Ciências Biológicas-bioquímica. Pós-doutorado em Imunologia e Biologia Molecular. Professor Associado do Departamento de Ciências Patológicas da Universidade Estadual de Londrina - UEL. Email: maewtuel@gmail.com
\end{abstract}


of IL-4 and the Th2 response. Furthermore, ginger inhibits translocation of the p65 subunit to the nucleus and inhibits phosphorylation of IkB - $\alpha$ complex, resulting in decreased levels of COX-2. We can conclude that components of ginger may act antagonistically on the effect of LPS activation of macrophages, leading to immunomodulatory and anti-inflammatory effects. The more likely affected signaling pathway is NF- $\kappa \mathrm{B}$ with involvement of MAP kinase.

Keywords: Zingiber officinale Roscoe. Gingerol. Shogaol. Inflammation

\section{Introdução}

O sistema imunológico é constituído por uma intrínseca rede de órgãos, células e moléculas, e tem por finalidade manter a homeostasia do organismo, combatendo as agressões em geral. A função imunológica tem sido conceitualmente dividida em imunidade inata e imunidade adaptativa. (MAHADY et al, 2003). Macrófagos possuem grande importância no sistema imune inato, no qual inicialmente funcionam como a primeira linha de defesa por iniciar a resposta inflamatória em resposta à exposição ao antígeno, participando da fagocitose e morte intracelular de microrganismos. Além disso, contribuem para o reparo de tecidos e agem como células apresentadoras de antígenos, que são requeridas para a indução de respostas imunes específicas (ABBAS; LICHTMAN, 2005). Desta forma, a inibição da ativação de macrófagos é uma das abordagens possíveis para a modulação do processo inflamatório.

Investigações têm sido realizadas a fim de caracterizar possíveis propriedades funcionais na prevenção e tratamento de várias doenças, como aterosclerose, diabetes, doenças cerebrais, inflamatórias crônicas e agudas e câncer. Neste contexto, tem se investido muito na avaliação das propriedades farmacêuticas e imunológicas de produtos naturais onde se destacam os extratos polifenólicos do gengibre (CRAIG; BECK, 1999; WEISBURGER, 1999).

O gengibre possui uma longa história quanto ao seu uso na medicina (VUTYAVANICH; KRAISARIN; RUANGSRI, 2001; AWANG, 1992), na dieta ou como erva medicinal, o qual é utilizado para tratar náuseas associada a gravidez ou ao movimento (BORRELLI et al., 2005; CHRUBASIK; PITTLER; ROUFOGALIS, 2005; VUTYAVANICH; KRAISARIN; RUANGSRI, 2001). O gengibre também é utilizado há séculos devido a suas propriedades anti-inflamatórias.

$\mathrm{O}$ gengibre, cujo nome científico é Zingiber officinale Roscoe, vem sendo utilizado tanto na medicina natural tradicional quanto contemporânea, e tem sido incluído nas farmacopeias no Reino Unido, Europa, China e Japão (WOHLMUTH et al., 2005). Diversos estudos indicam que os compostos encontrados no gengibre são eficazes no alívio dos sintomas de doenças inflamatórias crônicas como a colite ulcerosa, artrite reumatoide, entre outras doenças inflamatórias, devido sua ação anti-inflamatória, antitumoral, antioxidante, bactericida e antiviral (NAGENDRA CHARI et al, 2013). Além de sua atividade anti-inflamatória, o gengibre também apresenta propriedades carminativas, diaforéticas, antiespasmódicas, antieméticas, estimulantes da circulação periférica, anti-inflamatórias, estimulantes da digestão, hipoglicêmicas, colagogas e estomáticas, além da capacidade de inibir a síntese de prostaglandinas e a agregação plaquetária (NEWALL; ANDERSON; PHILLIPSON, 2002).

Portanto, esta revisão teve como objetivo descrever os componentes do gengibre bem como os mecanismos envolvidos em sua atividade anti-inflamatória, seu efeito antagônico sobre o processo inflamatório estimulado pelos lipopolissacarídeo (LPS) e a possível via de sinalização envolvida. 


\section{Gengibre e seus Componentes}

Tem sido descritos mais de 115 componentes do gengibre fresco e seco, identificados por diferentes processos analíticos (BENZIE; WACHTELGALOR, 2011; Harvey, 1981; HU etal., 2011; SHAO et al., 2010). A composição química do gengibre, segundo Newall et al. (2002), tem como principais componentes carboidratos e lipídeos, dentre eles ácidos graxos livres (ácido palmítico, ácido oléico, ácido linoleico), óleo-resina (homólogos de gingerol e zingerona), óleos voláteis (zingibereno, felandreno, canfeno), dentre outros. No entanto, a composição pode variar de acordo com a localização geográfica (países tropicais e subtropicais) em que foi cultivado (SMITH; ROBINSON, 1981). Os óleos essenciais de gengibre são compostos voláteis extraídos principalmente do gengibre com casca por processos de hidrodestilação (MAGALHÃES et al., 1997), segundo recomendado pela European Pharmacopoeia (2002). O gengibre na ausência da casca perde muitos componentes do óleo essencial, responsáveis pelo aroma. Já a óleoresina, obtida por extração com solventes (HE et al., 1998), contém, além dos constituintes aromáticos voláteis, os componentes não voláteis responsáveis pela pungência característica do gengibre (MAGALHÃES et al., 1997).

Cada produto do gengibre apresenta determinada característica físico-química que varia dependendo da origem e do método do preparo do material cuja escolha é determinada pela finalidade do seu uso (MAGALHÃES et al., 1997).

Os benefícios do gengibre para a saúde são principalmente devido à presença de compostos fenólicos, os quais são responsáveis por seu sabor pungente. São estes os gingeróis, de vários comprimentos de cadeia (n6 a n10), presentes em maior quantidade no gengibre fresco (BENZIE; WACHTEL-GALOR, 2011) e os shagois, a forma desidratada dos gingeróis, que são ainda mais pungentes e estão presentes no gengibre processado termicamente (ZHANG et al., 1994; HE et al., 1998).
Diversos componentes do gengibre têm sido amplamente estudados, sendo inúmeros os trabalhos publicados visando diferentes propriedades destas moléculas. Tais estudos incluem o papel dos compostos na atividade motora, efeito antipirético, analgésico (SUEKAWA et al., 1984), antioxidante (MASUDA et al., 2004), inibidor da angiogênese (KIM et al., 2005), inibidor de metástase de câncer de mama (LEE et al., 2008), antibacteriano (PARK; BAE; LEE, 2008), além das atividades imunomoduladoras e anti-inflamatórias que serão tratadas no presente trabalho. Há também estudos abordando suas propriedades físicas (DING et al., 1991) e farmacocinéticas em modelo animal (BHATTARAI; TRAN; DUKE, 2001) e humano (ZICK et al., 2008), os quais caminham na direção do desenvolvimento de medicamentos com tais compostos como princípio ativo.

Dentre as classes de componentes presentes no gengibre obtidas por extração (óleo-resina) e destilação (óleo-volátil), as principais encontram-se listadas no quadro 1. 
Quadro 1 - Principais componentes do gengibre.

\begin{tabular}{|l|l|}
\hline Fração óleo-resina & Fração óleo-volátil \\
\hline Paradols & $\beta$-phellandrene \\
\hline Gingerols & Camphene \\
\hline Isogingerols & Linalool \\
\hline Shogaols & Geranial \\
\hline [6]-isoshagaol & Zingiberene \\
\hline Gingerdiones & $\beta$-sesquiphellandiene \\
\hline Methoxy-gingerols & Neral \\
\hline Mono and di-acetoxy-gingerdiols & $\alpha$-bisabolene \\
\hline Dihydro-[6]-paradiols & $\alpha$-curcumene \\
\hline Diarylheptanoids & $\alpha$-famesene \\
\hline & $\alpha$-muurolene \\
\hline
\end{tabular}

Fonte: Adaptado de Joland et al., 2005 e Zhou; Deng; Xie, 2006

Dentre os componentes, destacam-se o 6-gingerol e o 6-shogaol e gingerdiones, que representam 3\% do óleo volátil, sendo os mais abundantes componentes da raiz (ZICK et al., 2008; VRIENS et al., 2008), motivo pelo qual são atualmente as moléculas mais exploradas individualmente (SAHA et al., 2014; KUMAR et al., 2014; DUGASANI, 2010; GAUS et al, 2009; TRIPATHI et al, 2007; ALI el al., 2007; KOH et al, 2009).

\section{Efeito Antagônico do Gengibre sobre o LPS}

Macrófagos tissulares possuem grande importância no sistema imune inato, em um primeiro momento funcionam como a primeira linha de defesa por montar uma resposta inflamatória a exposição ao antígeno, participando da fagocitose apresentadoras de antígenos, que são requeridas para a indução de respostas imunes específicas (ABBAS; LICHTMAN, 2005). Os macrófagos também são as principais células presentes na inflamação. A inibição da ativação de macrófagos é uma das abordagens possíveis para a inflamação de modulação.

Lipopolissacarídeo (LPS) também conhecido como endotoxina é uma molécula altamente tóxica e inespecífica derivada da membrana celular externa de bactérias Gram-negativas. Sua liberação ocorre quando a bactéria se multiplica ou quando é fagocitada e degradada pelas células de defesa (TUIN et al., 2006).

Tripathi et al. (2008), em um estudo utilizando cultura de macrófagos do peritônio de camundongos tratados com extrato de gengibre, observaram que o extrato diminuiu a ativação de macrófagos induzidos por LPS. Ele exerce um efeito inibitório sobre a produção de Interleucina 12 (IL -12), esta citocina estimula diretamente as células Natural Killers (NK) induzindo a produção de interferon gama (IFN- $\gamma$ ) que é importante para a ativação de macrófagos (MANETTI, et al, 1993). Além disso, observouse uma diminuição na expressão de moléculas coestimulatórias e de moléculas MHC classe II, o que diminui a atividade do macrófago como célula apresentadora de antígenos. Este comportamento é reforçado pela diminuição de interleucina 2 (IL-2), prejudicando dessa forma a ativação e proliferação de células T CD4+ em resposta ao aloantígeno. Além do mais, com relação a atividade fagocítica do macrófago, o composto 10-gingerdione, tem a capacidade de potencializá-la (KOH et al, 2009).

Interleucina 1 (IL-1) e o Fator de necrose tumoral alfa (TNF- $\alpha$ ) são citocinas pró inflamatórias produzidas principalmente por macrófagos e monócitos. A IL-1 possui efeito similar ao do TNF- $\alpha$, auxiliando na ativação de células T. A IL-1 pode induzir a secreção de IL-2 a partir do linfócito T ativado e esta citocina, por sua vez, está envolvida na ativação de células NK. (WILASRUSMEE et al., 2002).

O óleo volátil do gengibre inibe a secreção de IL-1 em macrófagos induzidos por LPS in vitro (ZHOU, DENG, XIE, 2006). Assim como o componente 6-gingerol diminui a secreção de IL-1, IL-12 e TNF$\alpha$ (TRIPATHI et al, 2007). O extrato de gengibre 
inibe a produção de IL-2 (WILASRUSMEE et al., 2002), e isso pode ter ocorrido devido a diminuição de IL-1 (ZHOU, DENG, XIE 2006).

Os macrófagos peritoneais de camundongos quando ativados com LPS produzem óxido nítrico (NO), que é um agente microbicida (CERQUEIRA; YOSHIDA, 2005). Esta produção é catalisada pela óxido nítrico sintase induzível (iNOS) que utiliza arginina como substrato produzindo $\mathrm{NO}$ e citrulina (LOWENSTEIN; PADALKO, 2004). Lee et al. (2009) verificaram que o 6-gingerol é capaz de diminuir a expressão de iNOS e de TNF- $\alpha$.

Koh et al. (2009) analisaram in vitro a ação dos componentes 6-shogaol, 1-de-hidro-[10]gingerdione e [10]-gingerdione na produção de NO. Eles verificaram que o 6-shogaol e o 1-dehidro-[10]-gingerdione foram capazes de diminuir significativamente a produção de NO pela inibição da expressão de iNOS.

A micróglia são células que fazem parte do sistema mononuclear fagocitário do sistema nervoso central (ABBAS; LICHTMAN, 2005). Há et al.(2012) investigaram o efeito do 6-shogaol na ativação da micróglia induzida por LPS em cultura de células microgliais primárias e observaram que o este composto inibiu a produção de $\mathrm{NO}$ e a expressão de iNOS.

A ciclooxigenase 2 (COX-2), é uma enzima, normalmente induzível, que participa da via do ácido araquidônico produzindo mediadores da inflamação como a prostaglandina E2 (PGE2) (ROBBINS \& COTRAN, 2010). Ha et al (2012) verificaram que o 6-shogaol inibiu a produção de PGE2 e citocinas pró inflamatórias como a IL-1 $\beta$ e o TNF- $\alpha$, através da regulação negativa de COX2, da subfamília de proteínas ativadas por mitógeno p38 (p38 MAPK) e do fator de transcrição NF-KB. Os mesmos autores também avaliaram o efeito do composto 12-Dehydrogingerdione (12-DHGD), sobre uma cultura de macrófagos ativados por LPS e observaram inibição na produção de NO, PGE2 e da citocina pró inflamatória IL-6. Além disso, constataram que o 12-DHGD inibiu o aumento da transcrição de iNOS e COX-2.

Os ReceptoresToll-like (TLR) desempenham papel fundamental na inflamação e no início da resposta imune subsequente atuando na resposta inata e adaptativa (OSPELTE; GAY, 2010). Os TLRs quando ativados, transmitem cascatas de sinais que culminam na fosforilação do $\operatorname{IkB} \alpha$ catalisado por IKKB, dando origem ao fator de transcrição kappa B (NF-kB), um potente indutor da transcrição de genes de citocinas pró-inflamatórias (RULAND; MAK, 2003). Lee et al. (2012) demonstraram que IKKB é alvo molecular do composto 1-Dehydro[10]-gingerdione (D10G), bloqueando sua ação catalítica e consequentemente suprimindo a expressão de NF-KB.

\section{Efeito Imunomodulador do Gengibre}

A via do ácido araquidônico é uma importante via imunomoduladora, uma vez que os eicosanoides (PGE2) e leucotrienos modulam de maneira direta o processo inflamatório e a resposta imune celular, inibindo a ação fagocítica dos macrófagos, a atividade das APCs (células apresentadoras de antígenos) e produção de citocinas próinflamatórias como a IL-2, IL-12 e induzindo a síntese de IL-10. Assim essas citocinas secretadas por diferentes grupos de células como Th2, células $\mathrm{T}$ reguladoras (Treg), macrófagos, linfócitos $\mathrm{B}$ e células dendrites modulam o sistema imunológico, promovendo o equilíbrio imunológico (MOORE et al, 2001; TRINCHIERI et al, 2001; HARIZI et al, 2002). Na década de 90, Srivastava e Mustafa demonstraram que a administração de gengibre em pacientes portadores de artrite reumatoide, promove a supressão tanto da via da cicloxigenase quanto dos metabólitos da lipoxigenase corroborando com outros estudos (TJENDRAPUTRA et al, 2001; SRIVAS, 1984).

Os extratos orgânicos à fresco e extratos secos de rizomas de gengibre possuem a capacidade de 
inibir o LPS bacteriano e possui capacidade de estimular a resposta imunológica (LIU; ZHU, 2002). Os extratos do gengibre dessa forma impedem o desencadeamento da resposta imune inicial impedindo, assim, a indução da PGE2 e diminuindo a produção de citocinas pró- inflamatórias como a interleucina (IL)-1B, IL-6 e o fator de necrose tumoral (TNF- $\alpha)$. Extratos contendo gingerols, shogaolsou gingerdiones demonstraram inibir fortemente a produção PGE2. No entanto, estudo que avaliaram somente a fração gingerdione, demonstraram que esse componente isolado apresenta um potencial inibitório da COX-2, porém não apresentam nenhuma ação sobre as citocinas pró-inflamatórias como a IL-1B e o TNF- $\alpha$ (HAN et al, 2013). Contudo, estudos que avaliaram a ação da fração total do gengibre, sobre a produção de PGE2, induzida por LPS, demonstraram, através da separação por cromatografia, que a fração com maior quantidade de gingerol e shogol apresentaram maior eficiência na inibição da PGE2 e de citocinas pró-inflamatórias, tais como a IL-1B e TNF-a, sendo atribuído a diminuição do PGE2 a fração gingerol e shogol e supressão de citocinas pró-inflamatórias a apenas a fração shogol do gengibre (LIU; ZHU, 2002; HA et al, 2012).

Diversos estudos mostram os efeitos imunomoduladores e antimicrobianos do gengibre, demonstrando que os óleos essenciais constituídos dos rizomas do gengibre, diminui a proliferação de bactérias e fungos como o Staphylococus sp. e Cândida albicans, porém, quando comparado o seu efeito com outros fitoterápicos como a citral e a curcumina, o gengibre não se mostrou tão eficiente em sua atividade microbicida. Foi verificado que extratos de gengibre estimulavam a secreção de IL-1 e IL-6, dependente da dose e do tempo. Tem sido demonstrado que o gengibre atua como um imune estimulante sobre as células de memória do sistema imunológico, uma vez que estimula a ativação e a diferenciação das células B (TAN; VANITHA, 2004).

Estudos com o óleo volátil de gengibre têm demonstrado que esse composto, possui atividade anti-inflamatória, podendo ser usado na clínica para o tratamento de artrite e outras desordens inflamatórias e dor crônica associada. Os resultados deste estudo indicaram que o óleo volátil de gengibre é capaz de modular linfócitos $\mathrm{T}$ inibindo a proliferação e diferenciação da subpopulação destas células em camundongo e humanos in vitro (WILASRUSMEE et al., 2002a). Esse resultado também foi verificado em outro estudo que demonstrou que o gengibre inibiu tanto mitose quanto a proliferação de linfócitos em camundongos (WILASRUSMEE et al., 2002b).

Em 2008, Ahui et al. descreveram o efeito do gengibre sobre a asma, em sistema murino. Demonstrando que extrato [6] e [8]-gingerol possui a capacidade de inibir a eosinofilia, diminuir a quantidade de mastócitos, inibir a liberação de IL-4 e a resposta Th2 responsável pelo desenvolvimento da doença. Estes resultados indicariam o gengibre como uma nova e promissora forma de tratamento uma vez que inibe a reação alérgica pulmonar e para outras doenças alérgicas de mesmo padrão.

Zhou et al. (2006) descreveram o efeito da imunomodulação do óleo volátil do gengibre, in vitro e in vivo em camundongos. Estes autores demonstraram que o óleo volátil do gengibre inibe significativamente a proliferação de linfócitos $\mathrm{T}$, diminuindo o número de células T helper. A ingestão de óleo volátil inibiu a secreção de IL-1 secretada por macrófagos além de diminuir a resposta de hipersensibilidade a 2,4-dinitro-1-fluorobenzeno em modelo in vivo, demonstrando que o gengibre influencia tanto a resposta mediada por células quanto a resposta não especifica a proliferação de células $\mathrm{T}$, sinalizando a importância no tratamento na doença inflamatória crônica e autoimune.

Lima (2003) verificou o potencial antiinflamatório do gengibre, através de modelos de pleurisia induzido por BCG em camundongos, onde demonstrou uma redução significativa da migração de células inflamatórias. Tem sido verificado em modelo in vitro, que no tratamento de câncer gástrico, o gengibre inibiu o crescimento do Helicobacter 
pylori, reduzindo consequentemente a patogênese da doença (MAHADY et al, 2003). Estudos in vivo, apresentaram efeito semelhante, uma vez que o tratamento com o extrato de gengibre contendo 6gingerol, reduziu H. pylori, reduziu tanto a inflamação aguda e crônica da mucosa e submucosa, bem como a degeneração das células epiteliais e erosão induzida por $H$. pylori. Uma vez que o extrato contendo 6-gingerol inibiu a atividade da ciclooxigenase-2, inibiu a resposta transcricional fator nuclear-kB em células Jurkat KBZ (linfócitos T humanos) e inibiu significativamente a produção de interleucina (IL)$1 \beta$, IL-6, IL-8 e TNF- $\alpha$ demonstrando assim que o extrato de gengibre pode ser útil para tratamento da inflamação induzida pelo $H$. pylori e a quimio prevenção do câncer gástrico (GAUS et al, 2009).

Assim, estes estudos demonstram que os componentes do gengibre possuem ação inibitória do processo inflamatório, por inibir a via da cicloxigenase, modular a resposta imunológica, através da supressão de citocinas pró-inflamatórias e consequentemente modular a via da resposta humoral mediada por células. $\mathrm{O}$ que pode explicar o potencial terapêutico do gengibre sobre diversas doenças.

\section{Efeito Anti-inflamatório do Gengibre: Possível Via de Sinalização}

Diversos compostos fenólicos são encontrados naturalmente em plantas, existem relatos de seus efeitos benéficos sobre inflamações e associação a propriedades antioxidantes (PRIOR; GAO, 2000). Os fenóis possuem a capacidade de capturar e neutralizar as espécies reativas de oxigênio (ERO) apresentando assim a capacidade anti-inflamatória por modular importantes vias de sinalização celular (KOBUCHI; VIRGILI; PACKER, 1999)

Extratos de gengibre apresentam compostos fenólicos como os shogaols, gingerols, entre outros (JOLAND et al., 2005). Choi et al. (2013) demonstraram em modelos de inflamação induzida por LPS administrado intraperitonialmente que o tratamento com o extrato seco do gengibre resulta na diminuição dos níveis plasmáticos de citocinas pró- inflamatórias como IFN $\gamma$ e IL-6. Foi verificado que o mesmo tratamento levou a uma diminuição da degradação do complexo

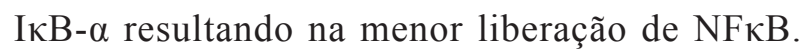
Também demonstraram uma diminuição na fosforilação de MAP quinases, como p38 ERK1/2 e JNK. Estes dois resultados corroboram então com a diminuição dos níveis plasmáticos das citocinas pró- inflamatórias bem como a inibição da expressão de iNOS e COX-2 (Choi et al., 2013).

Um outro estudo, utilizando um modelo de ratos submetidos a uma dieta hipercalórica, demonstrou que o tratamento com gengibre levou a uma diminuição na expressão de citocinas próinflamatórias em tecido hepático, como IL-6 e $\mathrm{TNF} \alpha$. In vitro demonstraram que o tratamento com o extrato resultava em uma menor atividade do $N F \kappa B$, e isto culminava com diminuição da expressão das citocinas IL-6 e TNF $\alpha$ (LI et al., 2012).

Em modelo experimental de asma induzidos pela imunização com ovoalbumina e posterior desafio com a mesma proteína, demonstrou que o tratamento com extrato do gengibre levou a diminuição da migração de leucócitos, sendo mais significante a redução da migração de eosinófilos. Também foi observado a redução de citocinas, como IL-5 INF $\gamma$, IL-4 e eotoxina, no soro, tecido pulmonar e lavado brônquicoalveolar. A diminuição de IL-4 parece estar relacionada com a diminuição da concentração de IgE (AHUI et al., 2008).

Estudos demonstraram que o 6-gingerol, assim como o extrato total, parece ter sua ação anti-inflamatória relacionado com a via do NFkB e MAP quinases. Aplicação tópica de o- tetradecanoyforbol-13-acetato (TPA) pode induzir aumento da expressão de COX- 
2 (CHUN et al., 2003; KUNDU et al., 2003). Kim e colaboradores em 2005 demonstraram que o pré-tratamento tópico com 6- gingerol em pele de camundongos parece diminuir os níveis de COX-2 induzida pelo TPA, mas sem efeitos sobre a COX-1. Estes mesmos autores demonstraram que este mesmo tratamento inibia a ativação do NFkB de diversas formas: inibindo a fosforilação do IkB- $\alpha$, inibindo a fosforilação e por conseguinte ativação da subunidade p65 da proteína culminando em uma menor translocação ao núcleo e ligação com o DNA. Porém os autores demonstraram que estes mecanismo parece estar relacionado com a interação com a via das MAP quinases com NFkB, em que a MAP quinase p38 ao ser inibida pelo 6-gingerol levava a não fosforilação do IkB- $\alpha$ e da subunidade p65. Culminando assim com a diminuição dos níveis de COX-2 (KIM et al., 2005).

Kim et al. (2005) demonstraram que o 6-gingerol reduziu os níveis de COX-2, inibiu a translocação da subunidade p65 para o núcleo, e também a inibição da fosforilação do complexo IkB- $\alpha$ em modelos de exposição de cultura de queratinócitos humanos e camundongos hairless à radiação UVB (KIM et al., 2007).

O 6-shogal, outro componente do gengibre, parece ter o mesmo efeito do 6-gingerol. Em cultura celular de micróglia estimulada com LPS, o composto 6-shogal levou a redução da ligação da subunidade p65 do NFkB ao DNA, bem como a diminuição da fosforilação do $\mathrm{IkB}$, explicando a redução da expressão da COX-2, de citocinas pró-inflamatórias como IL-1 $\beta$ e TNF- $\alpha$ (HA et al., 2012).

Por outro lado, Gan et al. (2013) demonstraram que não somente a modulação do NFkB parece estar relacionada com a modulação do processo inflamatório pelos compostos presentes no gengibre, mas também pela ativação da via do fator de transcrição Nfr2, que leva então a expressão de enzimas do sistema antioxidante, diminuindo o estresse oxidativo consequentemente modulando o processo inflamatório (GAN et al., 2013)

\section{Conclusão}

De acordo com o exposto nesta revisão, podemos concluir que componentes do gengibre atuam em diversas vias envolvidas no processo inflamatório e podem atuar antagonicamente sobre o efeito de ativação do LPS em macrófago, resultando em efeito imunomodulador e anti-inflamatório. A possível via de sinalização envolvida é a do fator de transcrição de citocinas pró-inflamatórias NFkB, com participação das MAP quinases e ativação do fator de transcrição Nrf2, relacionado a expressão de enzimas participantes das defesas antioxidantes.

\section{Referências}

ABBAS, A. K.; LICHTMAN, A. H. Imunologia Celular e Molecular, $5^{\circ}$ ed. Rio de Janeiro: Elsevier, 2005.

ALI, B.H.; BLUNDEN, G.; TANIRA, M.O.; NEMMAR, A. Some phytochemical, pharmacological and toxicological properties of ginger ZingiberofficinalisRoscoe:a review of recent research. Food Chemical Research in Toxicology, v. 46, p. 409-420, 2007.

AHUI, M. L. B.; CHAMPY, P.; RAMADAN, A.; VAN, L. P.; ARAUJO, L.; ANDRE, K. B.; DIEM, S.; DAMOTTE, D.; KATI-COULIBALY, S.; OFFOUMOU, M.A.; DY, M.; THIEBLEMONT, N.; HERBELIN, A. Ginger prevents TH2-mediated immune responses in a mouse model of airway inflammation. International Immunopharmacology, v. 8, n. 12, p. 1626-1632, 2008.

AWANG, D. V. C. "Ginger." Canadian Pharmaceutical Journal, v. 125, p. 309-311, 1992.

BENZIE, I. F. F.; WACHTEL-GALOR, S.The 
Amazing and Mighty Ginger. Herbal Medicine: Biomolecular and Clinical Aspects. 2. ed. Boca Raton (FL): CRC Press, cap.7, 2011.

BHATTARAI, S.; TRAN, V. H.; DUKE, C. C.The stability of gingerol and shogaol in aqueous solutions. Journal of Pharmaceutical Sciences, v. 90, n. 10, p. 1658-1664, 2001.

BORRELLI, F.; CAPASSO, R.; AVIELLO, G.; PITTLER, M. H.; IZZO, A. A. Effectiveness and safety of ginger in the treatment of pregnancyinduced nausea and vomiting. Obstetrics \& Gynecology, v. 105, n. 4, p. 849-856, 2005.

BRAHMBHATT, M.; GUNDALA, S. R.; ASIF, G.; SHAMSI, S. A.; ANEJA, R. Ginger phytochemicals exhibit synergy to inhibit prostate cancer cell proliferation. Nutritionand Cancer, v. 65, n. 2, p. 263-272, 2013.

CERQUEIRA, N. F.; YOSHIDA, W. B. Óxido nítrico: revisão. Acta Cirúrgica Brasileira, v. 17, n. 6, 2002.

CHOI, Y. Y.; KIM, M. H.; HONG, J.; KIM, S. H.; YANG, W. M. Dried Ginger (Zingiberofficinalis) Inhibits Inflammation in a LipopolysaccharideInduced Mouse Model. Evidence-Based Complementary and Alternative Medicine, 2013

CHRUBASIK, S.;PITTLER, M.H.;ROUFOGALIS, B. D. Zingiberisrhizoma: a comprehensive review on the effect and efficacy profiles. Phytomedicine, v. 12, n. 9, p. 684-701, 2005.

CHUN, K. S.; KEUM, Y. S.; HAN, S. S.; SONG, Y. S.; KIM, S. H.; SURH, Y. J. Curcumin inhibits phorbol ester-induced expression of cyclooxygenase-2 in mouse skin through suppression of extracellular signal-regulated kinase activity and NF-kappaB activation. Carcinogenesis, v. 24, n. 9, p. 15151524, 2003.

CRAIG, W.; BECK, L. Phytochemicals: Health Protective Effects. Canadian Journal of Dietetic Practice and Research, v. 60, n. 2, p. 78-84, 1999.

DING, G.H.; NAORA, K.; HAYASHIBARA,
M., KATAGIRI, Y.; KANO, Y.; IWAMOTO, K. Pharmacokinetics of [6]-gingerol after intravenous administration in rats. Chemical \& Pharmaceutical Bulletin, v. 39, n. 6, p. 1612-1614, 1991.

DUGASANIA, S.; PICHIKAC, M.R.; NADARAJAHC, V.D.; BALIJEPALLI C.M.K., TANDRAA, S.; KORLAKUNTAB, J.N. Comparative antioxidant and anti-inflammatory effects of [6]-gingerol,[8]-gingerol, [10]-gingerol and [6]-shogaol. Journal of Ethnopharmacology, v. 127 p. 515-520, 2010.

EUROPEAN DIRECTORATE FOR THE QUALITY OF MEDICINES \& HEARTH. Europea Pharmacopoeia, 4th ed. Strasbourg, p. 183-184, 2002.

GAN F.F.; LING H.; ANG X.; REDDY S.A.; LEE S.S.; YANG H.; TAN S.H.; HAYES J.D.; CHUI W.K.; CHEW E.H. A novel shogaol analog suppresses cancer cell invasion and inflammation, and displays cytoprotective effects through modulation of NF- $\mathrm{BB}$ and Nrf2-Keap1 signaling pathways. Toxicol Appl Pharmacol, v. 272, n. 3, p. 852-862, 2013.

GAUS, K.; HUANG, Y.; ISRAEL, D. A.; PENDLAND, S. L.; ADENIYI, B. A.; MAHADY, G. B. Standardized ginger (Zingiberofficinale) extract reduces bacterial load and suppresses acute and chronic inflammation in Mongolian gerbils infected with cagAHelicobacter pylori. Pharmaceutical Biology, v. 47, n. 1, p. 92-98, 2009.

HA S.K.; MOON E.; JU M.S.; KIM D.H.; RYU J.H.; OH M.S.; KIM S.Y. 6-Shogaol, a ginger product, modulates neuroinflammation: a new approach to neuroprotection. Neuropharmacology, v. 63, n. 2, p. 211-223, 2012.

HAN Y.A.; SONG C.W.; KOH W.S.; YON G.H.; KIM Y.S.; RYU S.Y.; KWON H.J.; LEE K.H. Anti-inflammatory effects of the Zingiberofficinale roscoe constituent12-dehydrogingerdione in lipopolysaccharide-stimulated Raw 264.7. cells. Phytother Research, v. 27, n. 8, p. 1200-1205, 2013. 
HANIADKA, R.; SALDANHA, E.; SUNITA, V.; PALATTY PL, FAYAD, R.; BALIGA, M. S. A review of the gastroprotective effects of ginger (Zingiberofficinale Roscoe). Food \& Function, v. 4 , n. 6, p, 845-855, 2013.

HARIZI, H.; JUZAN, M.; PITARD, V.; MOREAU, J. F.; GUALDE, N. Cyclooxygenase-2-issued prostaglandin $\mathrm{e}(2)$ enhances the production of endogenous IL-10, which down-regulates dendritic cell functions. The Journal of Immunology, v. 168, n. 5, p. 2255-2263, 2002a.

HARVEY, D. J. Gas Chromatographic And Mass Spectrometric Studies Of Ginger Constituents Identification of Gingerdiones And New Hexahydrocurcumin Analogues. Journal of Chromarography, v. 212, 75-84. 1981.

HE, X.; BERNART, M.W.; LIAN, L.; LIN, L. Highperformance liquid chromatography-electrospray mass spectrometric analysis of pungent constituents of ginger. Journal of Chromatography A, v. 796, p. 327-334. 1998.

HU, J.; GUO, Z.; GLASIUS, M.; KRISTENSEN, K.; XIAO, L.; XU, X. Pressurized liquid extraction of ginger (Zingiberofficinale Roscoe) with bioethanol: An efficient and sustainable approach. Journal of Chromatography A, v. 1218, p. 5765- 5773, 2011.

JEENA, K., LIJU, V. B.; VISWANATHAN, R.; KUTTAN, R. Antimutagenic Potential and Modulation of Carcinogen-Metabolizing Enzymes by Ginger Essential Oil. Phytotherapy Research, 2013.

JEENA, K.; LIJU, V. B.; KUTTAN R. Antioxidant, anti-inflammatory and antinociceptive activities of essential oil from ginger. Indian Journal of Physiology and Pharmacology, v. 57, p. 51-62, $2013 \mathrm{a}$.

JOLAND, S. D.; LANTZ, R.C., CHEN, G. J.; BATES, R. B.; TIMMERMANN, B. N. Commercially processed dry ginger (Zingiberofficinale): Composition and effects on LPS-stimulated PGE2 production. Phytochemistry, v. 66, p. 1614-1635, 2005.
KIM, E.C.; MIN, J.K.; KIM, T.Y.; LEE, S.J.; YANG, H.O.; HANB, S.; KIME, Y.; KWONA, Y. [6]-Gingerol, a pungent ingredient of ginger, inhibits angiogenesis in vitro and in vivo. Biochemical and Biophysical Research Communications, v. 335, n. 2, p. 300-308, 2005.

KIM, J. K.; KIM, Y.; NA K. M.; SURH Y. J.; KIM T. Y. [6]-Gingerol prevents UVB-induced ROS production and COX-2 expression in vitro and in vivo. Free Radical Research, v .41, n. 5, p. 603-614, 2007.

KIM, S. O.; KUNDU, J. K.; SHIN, Y. K.; PARK, J. H.; CHO, M. H.; KIM, T. Y.; SURH Y. J. [6]-Gingerol inhibits COX-2 expression by blocking the activation of p38 MAP kinase and NF-kappaB in phorbol esterstimulated mouse skin. Oncogene, v. 24, n. 15, p. 2558-2567, 2005.

KOBUCHI, H.; VIRGILI, F.; PACKER, L. Assay of inducible form of nitric oxide synthase activity: effect of flavonoids and plant extracts. Methods in Enzymology, v. 301, p. 504-513, 1999.

KOH, E. M.; KIM, H. J.; KIM, S.; CHOI, W. H.; CHOI, Y. H.; RYU, S. Y.; KIM, Y. S.; KOH, W. S.; PARK, S. Y. Modulation of macrophage functions by compounds isolated from Zingiberofficinale. Planta Médica, v. 75, n. 2 , p. 48-51, 2009.

KUMAR, N. V.; MURTHY, P.S.; MANJUNATHA, J.R.; BETTADAIAH, B.K. Synthesis and quorum sensing inhibitory activity of key phenolic compounds of ginger and their derivatives. Food Chemistry, v. 159, p. 451-457, 2014.

KUNDU, J. K.; NA, H. K.; CHUN, K. S.; KIM, Y. K.; LEE, S. J.; LEE, S. S.; LEE, O. S.; SIM, Y. C.; SURH, Y. J. Inhibition of phorbol ester-induced COX-2 expression by epigallocatechingallate in mouse skin and cultured human mammary epithelial cells. The Journal of Nutrition, v. 133, 2003.

LEE, H.S.; SEO, E.Y.; KANG, N.E.; KIM, W.K. [6]-Gingerol inhibits metastasis of MDA-MB-231 human breast cancer cells. The Journal of Nutritional Biochemistry, v. 19, n. 5, p. 313-319, 2008. 
LEE, J.; OH, H. Ginger as an antiemetic modality for chemotherapy-induced nausea and vomiting: a systematic review and meta-analysis. Oncology Nursing Forum, v. 40, n. 2, p. 163-70, 2013.

LEE, H, Y., PARK, S, H., LEEL, M., KIM, HYE-JIN., RYU, S, Y., KIM, N, D., HWANG, B, Y., HONG, J, T., HAN, SANG-BAE., KIM, Y. 1-Dehydro-[10]gingerdione from ginger inhibits IKK $\beta$ activity for NF-k $\beta$ activation and suppresses NF-k $\beta$-regulated expression of inflammatory genes. British Journal of Pharmacology, 2012.

LEE, T. Y.; LEE, K.. C.; CHEN, S. Y.; CHANG, H. H. 6-Gingerol inhibits ROS and iNOS through the suppression of PKC-alpha and NF-kappaB pathways in lipopolysaccharide-stimulated mouse macrophages. Biochemical and Biophysical Research Communications, v. 382 ,p. 134-139, 2009.

LI, X. H.; MCGRATH, K. C.; NAMMI, S.; HEATHER, A. K.; ROUFOGALIS, B. D. Attenuation of liver pro-inflammatory responses by Zingiberofficinale via inhibition of NF-kappa B activation in high-fat diet-fed rats. Basic \& Clinical Pharmacology \& Toxicology, v. 110, n. 3, p. 238-244, 2012.

LIMA, M. O. Efeito do tratamento com extratos hidroalcoólios e cetônicos do rizoma de Zingiberofficinale no modelo esperimental de pleurisia induzida por BCG em camundongos, 70p. 2003. Dissertação (Mestrado em ciências biológicas)- Instituto de pesquisa e Desenvolvimento, Universidade do Vale do Paraíba, São José dos campos, 2003.

LIU H.; ZHU Y. Effect of alcohol extract of Zingbenofficinale rose on immunologic function of mice with tumor. Wei Sheng Yan Jiu, v. 31, n. 3, p. 208-20, 2002.

LOWENSTEIN, C. J.; PADALKO, E. iNOS (NOS2) at a glance.Journal of Cell Science, v. 117, p. 28652867, 2004.

MAGALHÃES, M. T.; KOKETSU, M.; GONÇALVES, S. L.; CORNEJO F.E.P.; MARQUES
L. M. R. Gengibre (zingiberofficinaleroscoe) brasileiro: aspectos gerais, óleo essencial e oleoresina. parte 2 - secagem, óleo essencial e oleoresina. Food Science and Technology, v. 17, n. 2, p. 132-136, 1997.

MAHADY, G. B.; PENDLAND, S. L.; YUN, G. S.; LU, Z. Z.; STOIA, A. Ginger (Zingiberofficinale Roscoe) and the gingerols inhibit the growth of CagA+ strains of Helicobacter pylori. Anticancer Research, v.23, p. 3699-3702, 2003.

MANETTI, R.; PARRONCHI, P.; GIUDIZI, M. G.; PICCINNI, M. P.; MAGGI, E.; TRINCHIERI, G.; ROMAGNANI, S. Natural killer cell stimulatory factor (interleukin 12 [IL-12]) induces T helper type 1 (Th1)-specific immune responses and inhibits the development of IL-4-producing Th cells. The Journal of Experimental Medicine, v. 177, n. 4, p. 1199-1204, 1993.

MARX, W. M.; TELENI, L.; MCCARTHY, A. L.; VITETTA, L.; MCKAVANAGH, D.; THOMSON, D.; ISENRING, E. Ginger (Zingiberofficinale) and chemotherapy-induced nausea and vomiting: a systematic literature review. Nutrition Reviews, v.71, n. 4, p. 245-254, 2013.

MASCOLO, N.; JAIN, R.; JAIN, S. C.; CAPASSO, F. Ethnopharmacologic investigation of ginger (Zingiberofficinale). Journal of Ethnopharmacology, v. 27, p. 129-140, 1989.

MASUDA, Y., KIKUZAKI, H.; HISAMOTO, M.; NAKATANI, N. Antioxidant properties of gingerol related compounds from ginger. Bio Factors, v. 21, p. 293-296, 2004.

MOORE, K.W; DE WAAL-MALEFYT R.; COFFMAN, R. L.; O'GARRA, A. Interleukin-10 and the interleukin-10 receptor. Annual Review of Immunology,v. 19, p. 683-765, 2001

NAGENDRA CHARI K.L.; MANASA D.; SRINIVAS P.; SOWBHAGYA H.B. Enzymeassisted extraction of bioactive compounds from ginger (Zingiberofficinale Roscoe). Food Chemistry, v. 15 n. 139(1-4), p. 509-514, 2013. 
NEWALL, C. A.; ANDERSON, L. A.; PHILLIPSON J. D. Tr Mirtes Frange de Oliveira Punheiro. Plantas Medicinais: guia para profissional de saúde. São Paulo: Editorial Premier, 2002.

OSPELT, C.; GAY, S. TLRs and chronic inflammation. The International Journal of Biochemistry \& Cell Biology, v. 42, p. 495-505, 2010.

PALATTY, P. L.; HANIADKA, R.; VALDER, B.; ARORA, R.; BALIGA, M. S. Ginger in the prevention of nausea and vomiting: a review. Critical Reviews in Food Science and Nutrition, v. 53, n. 7, p. 659-669, 2013.

PARK, M.; BAE, J.; LEE, D. S. Antibacterial activity of [10]-gingerol and [12]-gingerol isolated from ginger rhizome against periodontal bacteria. Phytotherapy Research, v. 22, p. 1446-1449, 2008.

PHANG, C. W.; MALEK, S. N.; IBRAHIM, H. Antioxidant potential, cytotoxic activity and total phenolic content of Alpiniapahangensis rhizomes. BMC Complementary and Alternative Medicine, v. 13, p. 243, 2013.

PRIOR, R. L.; CAO, G. Flavonoids: diet and health relationships. Nutrition in Clinical Care, v. 3, n. 5, p. 279-288, 2000.

RAMADAN, G.; EL-MENSHAWY, O. Protective effects of ginger-turmeric rhizomes mixture on joint inflammation, atherogenesis, kidney dysfunction and other complications in a rat model of human rheumatoid arthritis. International Journal of Rheumatic Diseases, v. 16, n. 2, p. 219-229, 2013.

ROBBINS \& COTRAN. Patologia: Bases patológicas das doenças, $8^{\mathrm{a}}$ ed. Rio de Janeiro: Elsevier, 2010

RULAND, J.; MAK, T. W. Transducing signals from antigen receptors to nuclear factor $\mathrm{kB}$. Immunol Review 193: 93-100, 2003.

SAHA, A.; BLANDO, J.;SILVER, E.;BELTRAN, L.; SESSLER, J.;DIGIOVANNI, J. 6-Shogaol from Dried Ginger Inhibits Growth of ProstateCancer Cells Both In Vitro and In Vivo through Inhibition ofSTAT3 and
NF-kB Signaling. Cancer Prevention Research; v. 7, n.6, p. 627-38, 2014.

SHAO, X.; LV, L.; PARKS, T.; WU, H.; HO, C.; SANG, S. Quantitative analysis of ginger components in commercial products using liquid chromatography with electrochemical array detection. Journal of Agricultural and Food Chemistry, v. 58, n. 24, p. 12608-12614, 2010.

SMITH, R. M.; ROBINSON, J. M. The Essential Oil Of Ginger From Fiji. Phytochemistry, v. 20, p. 203206, 1981.

SRIVAS, K. C. Effects of aqueous extracts of onion, garlic and ginger on platelet aggregation and metabolism of arachidonic acid in the blood vascular system: in vitro study. Prostaglandins, Leukotrienes and Medicine, v. 13, n. 2, p. 227-235, 1984.

SRIVASTAVA, K. C.; MUSTAFA, T. Ginger (Zingiberofficinale)in rheumatismandmusculoskeletal disorders. Medical Hypotheses, v. 39, n. 4, p. 342-348, 1992.

SUEKAWA, M.; ISHIGE, A.; YUASA, K.; SUDO, K.; ABURADA, M.; HOSOYA, E. Pharmacological studies on ginger. I. Pharmacological actions of pungent constitutents, (6)-gingerol and (6)-shogaol. Journal Pharmacobiodyn, v. 7, n. 11, p. 836-848, 1984.

TAN, B. K.; VANITHA J. Immunomodulatory and antimicrobial effects of some traditional chinese medicinal herbs: a review. Current Medicinal Chemistry, v. 11, n. 11, p. 1423-1430, 2004.

TJENDRAPUTRA, E.; TRAN, V. H.; LIUBRENNAN, D.; ROUFOGALIS, B. D.; DUKE, C. C. Effect of ginger constituents and synthetic analogues on cyclooxygenase-2 enzyme in intact cells. Bioorganic Chemistry, v. 29, n. 3, p. 156-163, 2001.

TRINCHIERI, G. Regulatory role of T cells producing both interferon gamma and interleukin 10 in persistent infection. The Journal of Experimental Medicine, v. 194, 2001.

TRIPATHI, S.; MAIER, K. G.; BRUCH, D.; KITTUR, 
D. S. Effect of 6-gingerol on proinflammatory cytokine production and costimulatory molecule expression in murine peritoneal macrophages. Journal of Surgical Research, v. 138,n. 2, p. 209-213, 2007.

TRIPATHI, S.; BRUCH, D.; KITTUR, D.S. Ginger extract inhibits LPS induced macrophage activation and Function. BMC Complementary and Alternative Medicine, v. 8, 2008.

TUIN,A.; VLAG,A.H.V.D., VOENEN-WEEMAES, A. M. M. A. V., MEIJER, D. K. F., POELSTRA, K. On the role and fate of LPS-dephosphorylating activity in the rat liver. American Journal of PhysiologyGastrointestinal and Liver Physiology, v. 290, p. 377385, 2006.

VRIENS, J.; NILIUS, B.; VENNEKENS, R. Herbal compounds and toxins modulating TRP channels. CurrNeuropharmacol, v. 16, p. 79-96, 2008.

VUTYAVANICH, T.; KRAISARIN, T.; RUANGSRI, R. A. Ginger for nausea and vomiting in pregnancy: randomized, double-masked, placebo-controlled trial. Obstetrics \& Gynecology, v. 97, p. 577-582, 2001.

WEISBURGER, J. H. Worldwide optimal nutrition, disease prevention, and health promotion. Nutrition, v. 15, p. 949-50, 1999.

WILASRUSMEE, C., SIDDIQUI, J., BRUCH, D., WILASRUSMEE, S., KITTUR, S., KITTUR, D.S. In vitro immunomodulatory effects of herbal products. The American Surgeon, v. 68, p. 860-864, 2002 b.

WILASRUSMEE, C.; KITTUR, S.; SIDDIQUI, J.; BRUCH, D.; WILASRUSMEE, S.; KITTUR, D. S. In vitro immunomodulatory effects of ten commonly used herbs on murine lymphocytes. Journal of Alternative and Complementary Medicine, v. 8, n. 4, p. 467-475, 2002a.

WOHLMUTH, H.; LEACH D. N.; SMITH, M. K.; MYERS, S. P. Gingerol content of diploid and tetraploid clones of ginger (Zingiberofficinale Roscoe). Journal of Agricultural and Food Chemistry, v. 53, n. 14, p. 5772-5778, 2005.
ZHANG, X.; IWAOKA, W. T.; HUANG, A. S.; NAKAMOTO, S. T.; WONG, R. J. Gingerol Decreases after Processing and Storage of Ginger. Journal of Food Science, v. 59, p. 1338-1340, 1994.

ZHOU, H. L.; DENG, Y. M.; XIE, Q. M. The modulatory effects of the volatile oil of ginger on the cellular immune response in vitro and in vivo in mice. Journal of Ethnopharmacology, v. 105, p. 301-305, 2006.

ZICK, S. M.; DJURIC, Z.; RUFFIN, M. T.; LITZINGER, A. J.; NORMOLLE, D. P.; ALRAWI, S.; FENG, M. R.; BRENNER, D. E. Pharmacokinetics of 6-Gingerol, 8-Gingerol, 10-Gingerol, and 6-Shogaol and Conjugate Metabolites in Healthy Human Subjects. Cancer Epidemiology, Biomarkers \& Prevention, v. 17, n. 8, p. 1930-1936, 2008.

Recebido em: 27 nov. 2013

Aceito em: 03 jun. 2014 
